\title{
The Influence of Boundary Conditions on Modulus Measurement in Uniaxial Compression Tests
}

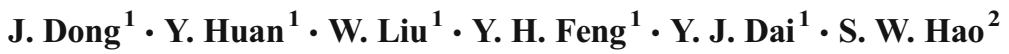

Received: 30 March 2016 / Accepted: 13 February 2017 /Published online: 24 February 2017

(C) The Society for Experimental Mechanics, Inc 2017

\begin{abstract}
There is distinct deviation between the intrinsic and measurement value of Young's modulus in uniaxial compression tests. The key influence factor has always been owned to the friction between compression platens and specimen. However, we found this is not true by Finite element analysis (FEA) and experiments. FEA results show that the friction only makes a major influence on the plastic behavior, resulting in a barreling deformation, rather than on the elastic deformation behavior of specimen. In fact, the oblique contact between compression plate and specimen will severely influence the homogeneity of the stress field in specimen, and result in the deviation of the measured modulus value. This study can well interpret the relevant provision in ASTM E9-09 that only slim specimens (with aspect ratio $\geq 8$ ) are suitable for the compression modulus tests.
\end{abstract}

Keywords Compression tests $\cdot$ Modulus $\cdot$ Finite elements analysis $($ FEA) $\cdot$ Oblique contact $\cdot$ Friction

\section{Introduction}

Uniaxial compression tests are widely used to characterize the materials' response under compressive loading. It has advantages in specimen preparation and experiment operation, along with a simple mechanical model. For understanding the behavior of materials under large plastic strains, compression test is also more appropriate than tensile test because the latter is

Y. Huan

huany@1nm.imech.ac.cn

1 State Key Laboratory of Nonlinear Mechanics (LNM), Institute of Mechanics, Chinese Academy of Sciences, Beijing 100190, China

2 School of Civil Engineering and Mechanics, Yanshan University, Qinhuangdao 066004, China limited by necking [1]. Especially, the compression test is easier for metallic glass to produce the plasticity than tensile test [2-5]. In addition, compression tests are necessary for characterizing the mechanical behavior of anisotropic materials and bimodular materials [6-8], such as highly textured materials that deform by twinning as two separate crystals share some of the same crystal lattice points in a symmetrical manner[9].

However, the drawback of compression tests is that the tested modulus is different from the intrinsic value for middle and small aspect ratio specimens. It should be noted that the modulus measurement value closely depends on the aspect ratio of specimen. In order to acquire the true value of modulus as reliably as possible, a high aspect ratio $(\geq 8)$ is recommended by ASTM E9-09 [10]. The chief culprit has been owned to the friction between the specimen and the compression plates, which leads to an inhomogeneous stress field and destroys the assumption of one-dimensional stress. A powerful proof is that the specimens of elastic-plastic materials usually show a barreling shape after compression. The inhomogeneous stress field produced by the friction has been investigated through theory analyses and experiments [11-15]. However, Wiegels and Herbertz [16] conducted a comprehensive experimental study of the compression tests and found that the influence of friction on the stress homogeneity was small. The chief culprit of the modulus measurement deviation is still not determined.

In this paper, the influences of boundary conditions in compression tests are investigated using Finite element analysis and experiments. With regard to the reason of modulus measurement deviation, the chief culprit is the oblique contact between compression plate and the specimen, rather than the friction.

\section{Experiments}

Three kinds of common engineering materials, Steel 1045, Brass C28000 and Cast iron No.20, were chosen for compression testing using a MTS 810 testing machine. Specimens with diameter 
a

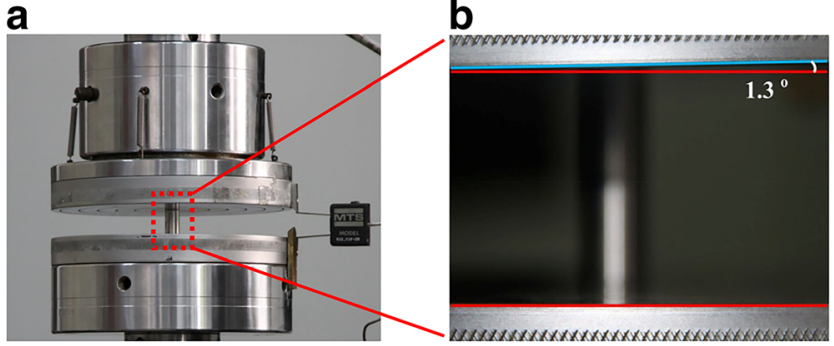

Fig. 1 (a) Experimental set up. (b) The angle of the compression plate with respect to the horizontal plane is measured as $1.3^{\circ}$

of $10 \mathrm{~mm}$ and length of $30 \mathrm{~mm}$ were compressed between two plates and the deformation was measured by a COD (Crack Open Displacement) displacement sensor, as shown in Fig. 1(a). The angle of the compression plate with respect to the horizontal plane is measured as $1.3^{\circ}$ shown in Fig. 1(b). The tests were conducted at room temperature with a strain rate of $1 \times 10^{-4} \mathrm{~S}^{-1}$. At least three specimens for each kind of material were tested to verify the reliability of the results.

The tested true stress-strain curves of steel 1045 are shown in Fig. 2. One can see that the initial segment is nonlinear. The tested modulus, which is calculated from the slope of the linear segment, is lower than intrinsic value (Fig. 2(b)). It is believed that this phenomenon is caused by the boundary conditions. In fact, the actual contact surface between the compression plate and the specimen is neither ideally smooth nor absolutely parallel. Thus the test results will be inevitably influenced by the friction and the oblique contact between the compression plate and specimen.

\section{Finite Element Analysis}

Finite element analysis was carried out to evaluate the influences of boundary conditions in compression tests. In order to investigate the effects of friction and oblique contact respectively, models with different boundary conditions were established.

A friction model is established using 2D axisymmetric model with only friction being applied on the ends of specimen of Steel
1045, shown in Fig. 3(a). The upper and lower plates are absolutely parallel to the specimen surface. The simulation results show that most part of the material is in the uniform stress field, while only a tiny part material near the boundaries is affected by friction. The simulation stress-strain curve is linear from the initial point to yield point. The slope is close to the intrinsic modulus, but bigger than the tested value. Obviously, the two curves are different, shown in Fig. 3(b). This phenomenon indicates that the influence of friction is not as significant as previously conceived. In addition, a barreling shape deformation can been seen in the later stage, shown in Fig. 3(c). Thus, it can be determined that the friction only has a main influence on the plastic deformation, rather than on the modulus measurement.

In fact, the plane contact is not absolute in the beginning of the compression test. In order to investigate the influence of oblique contact, a contact model is established using 3D model with the upper compression plate being inclined about $1.3^{\circ}$ and the friction being still taken into account. As shown in Fig. 4(a), a significant difference is found that the stress field becomes highly inhomogeneous. A high stress zone is generated near the contact surface in the beginning of compression. The initial arc-shaped segment of the stress-strain curve just corresponds to the oblique contact process, as shown in Fig. 4(b). Along with the load increasing, the high stress zone extends gradually towards the middle part of the specimen. After the specimen and plate are fully contacted, the stress-strain curve becomes linear and the stress field also becomes uniform correspondingly. However, the material near the contact surface becomes plastic earlier than other part of the specimen, leading to the slope of the linear segment of stress-strain curve lower than that of an ideal elastic specimen. Thus the tested compression modulus is also lower than intrinsic value.

The compression test in fact starts with the oblique contact between compression plate and specimen, rather than with the absolute plane contact. The local plastic zone generated by the oblique contact is the main factor causing the compression modulus lower than the intrinsic value. The similar result is also obtained by the FEA of Brass C28000 and Cast iron No.20, as shown in Fig. 5.
Fig. 2 (a) Compression engineering stress-strain curves of steel 1045. (b) The initial segment of the test curve is nonlinear and the tested modulus is lower than intrinsic value
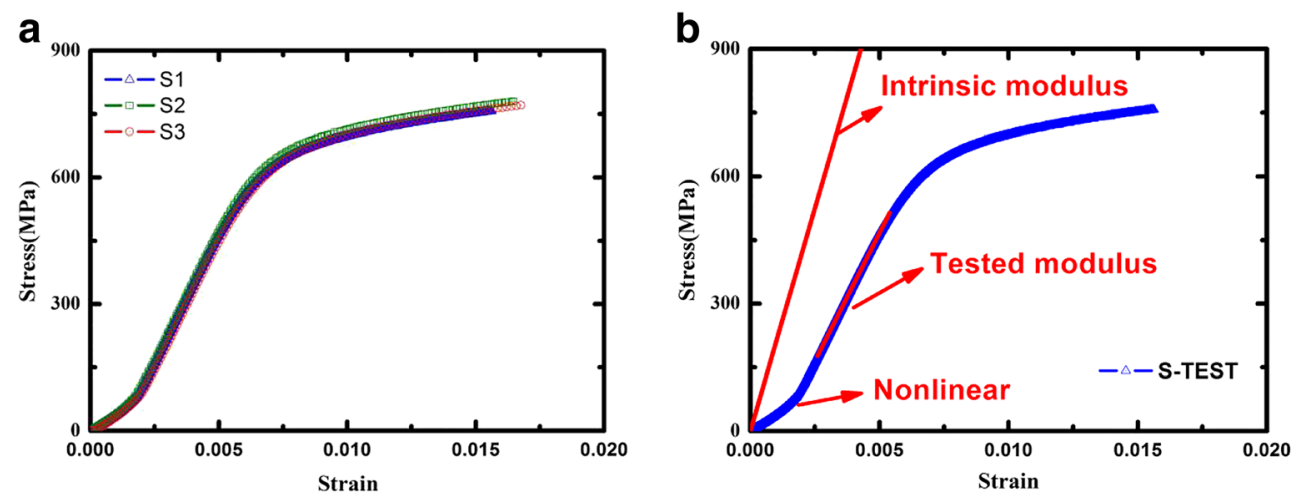
Fig. 3 Simulation results of Steel 1045 in Friction model, where only the effect of friction is considered: (a) Most of the stress field is nearly uniform; (b) The simulation curve deviates from the test curve significantly; (c) The barreling shape deformation occurs after large strain compression

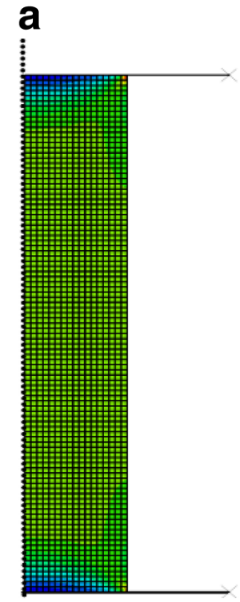

b
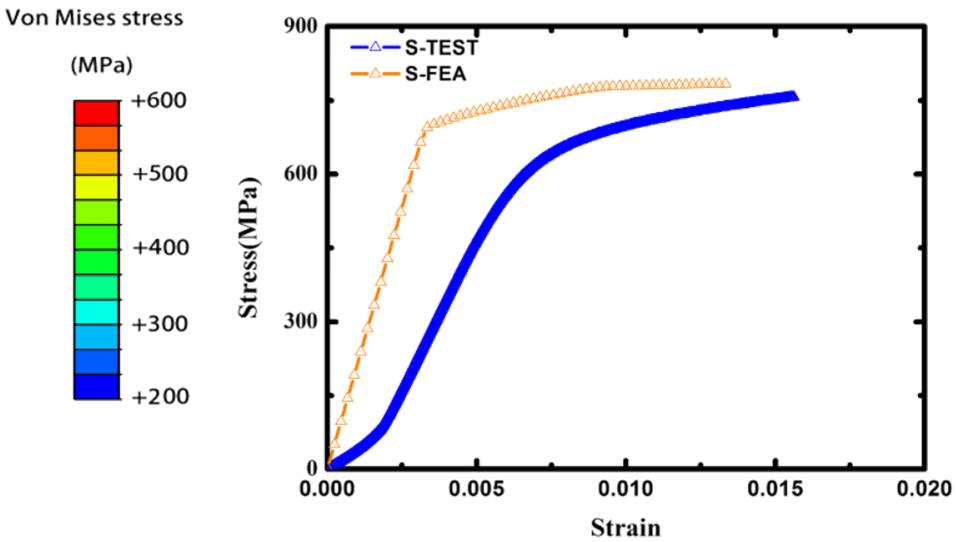

C

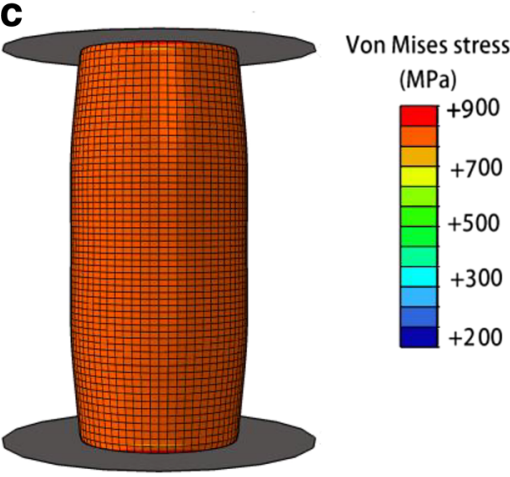

Strain

s

00

500

300

200
Fig. 4 Simulation results of Steel 1045 in contact model, where both the friction and the oblique contact are included: (a) The stress field is highly

inhomogeneous caused by the oblique contact; (b) The simulation curve of contact model can coincide well with testing curve a

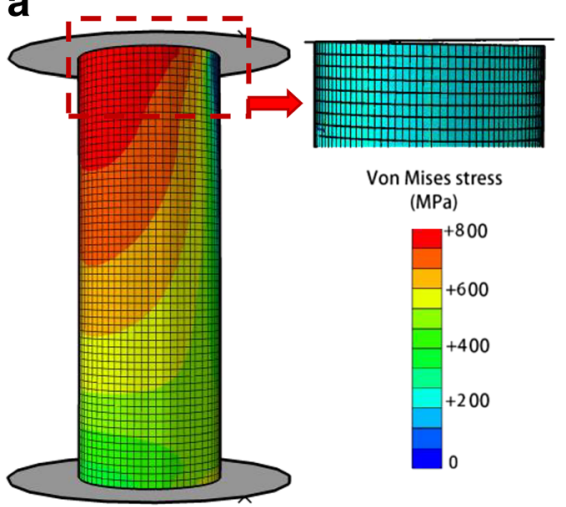

a

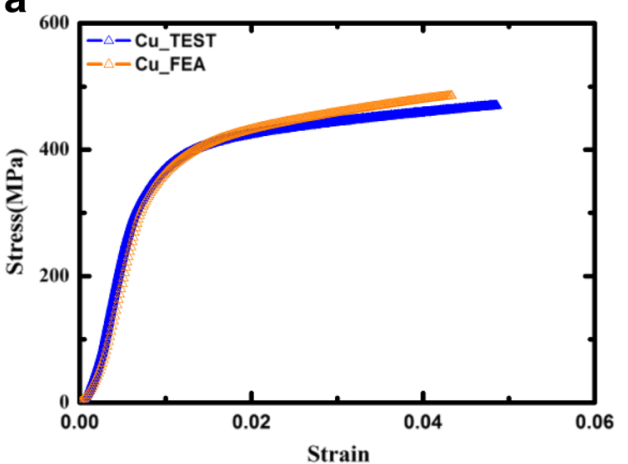

b

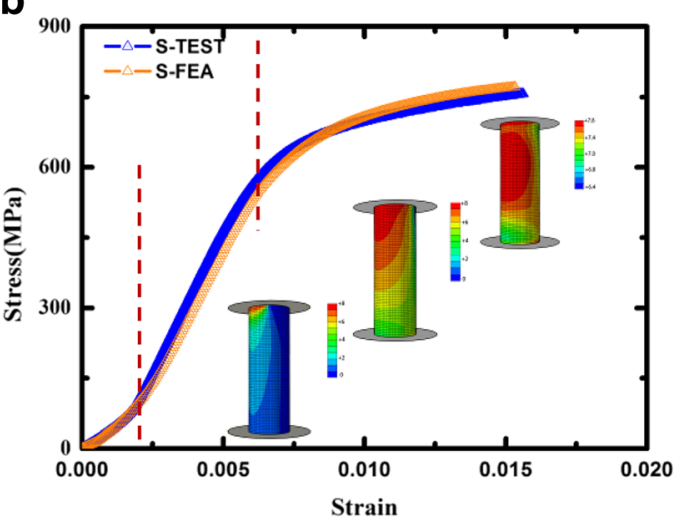

b

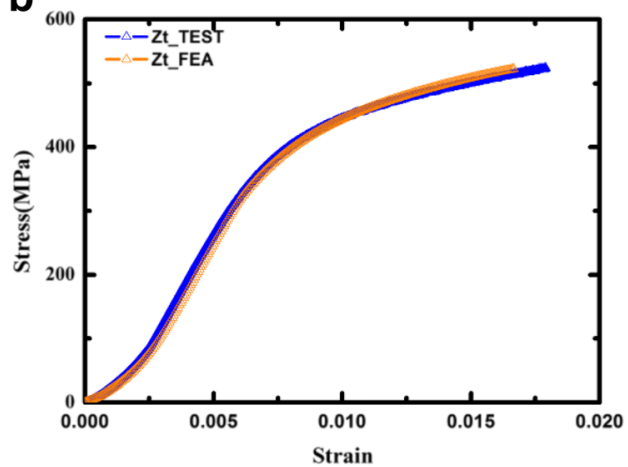

Fig. 5 The simulation curve of Brass C28000 (a) and (b) Cast iron No.20 in contact model also coincide well with the testing curve 


\section{Conclusion}

In this study, the boundary conditions of compression tests are investigated using Finite element analysis and experiments. By comparing the simulation results with the test stress-strain curves, following conclusions can be obtained.

(1) The friction has a poor influence on the modulus measurement. It only makes a significant influence on the plastic behavior, which causes a barreling shape after deformation.

(2) The oblique contact is the key factor influencing the modulus measurement. The initial arc-shaped segment of stress-strain curve corresponds to the initial contacting process between the specimen and the plate, which starts from point contact to plane contact.

(3) The local plastic zone generated by oblique contact is the culprit that causes the tested modulus lower than the intrinsic value.

Acknowledgements This work is supported by the National Natural Science Foundation of China (Grant No. 11372323, 11302231), National Basic Research Program of China (Grant No. 2013CB834100) and CAS Key Technology Talent Program.

\section{References}

1. Dieter GE (1986) Mechanical metallurgy. McGraw-Hill, New York

2. Hufnagel TC, Jiao T, Li Y et al (2002) Deformation and failure of Zr57Ti5Cu20Ni8Al1 bulk metallic glass under quasi-static and dynamic compression. J Mater Res 17:1441-1445
3. Schuster BE, Wei Q, Hufnagel TC et al (2008) Size-independent strength and deformation mode in compression of a Pd-based metallic glass. Acta Mater 56:5091-5100

4. Gu J, Song M, Ni S et al (2014) Improving the plasticity of bulk metallic glasses via pre-compression below the yield stress. Mater Sci Eng A 602:68-76

5. Shahabi HS, Scudino S, Kaban I et al (2015) Structural aspects of elasto-plastic deformation of a $\mathrm{Zr}$-based bulk metallic glass under uniaxial compression. Acta Mater 95:30-36

6. Jones RM (1976) Apparent flexural modulus and strength of multimodulus materials. J Compos Mater 10:342-354

7. Medri G (1982) A nonlinear elastic model for isotropic materials with different behavior in tension and compression. J Eng Mater Technol 104:26-28

8. Sun JY, Zhu HQ, Qin SH et al (2010) A review on the research of mechanical problems with different moduli in tension and compression. J Mech Sci Technol 24:1845-1854

9. Charalambides MN, Goh SM, Lim SL et al (2001) The analysis of the frictional effect on stress-strain data from uniaxial compression of cheese. J Mater Sci 36:2313-2321

10. ASTM E9-09 (2009) Standard test methods of compression testing of metallic materials at room temperature. ASTM International, West Conshohocken, http://www.astm.org

11. Lindley PB (1979) Compression moduli for blocks of soft elastic material bonded to rigid end plates. J Strain Anal Eng Des 14:12-16

12. Chau K (1997) Young's modulus interpreted from compression tests with end friction. J Eng Mech 123:1-7

13. Watanabe S (1998) Discussion: young's modulus interpreted from compression tests with end friction. J Eng Mech 124:1170-1174

14. Williams JG, Gamonpilas C (2008) Using the simple compression test to determine young's modulus poisson's ratio and the coulomb friction coefficient. Int J Solids Struct 45:4448-4459

15. Henke T, Bambach M, Hirt G et al (2011) Experimental uncertainties affecting the accuracy of stress strain equations by the example of a HenselSpittel approach. AIP Conf Proc 1353:71-76

16. Wiegels H, Herbertz R (1979) Cylinder upsetting test with high friction for the determination of the yield stress. Stahl Eisen 99: $1380-1390$ 